\title{
A Modeling Method of Power System Based on Brittleness Factor and Brittleness Correlation Factor
}

\author{
Xiao Lyu* , Dan Lyu, Yuge Yang \\ School of Electrical \& Automation Engineering, Nanjing Normal University, Nanjing, China \\ *Corresponding author: nnulvxiao@126.com
}

Received June 04, 2019; Revised July 13, 2019; Accepted July 24, 2019

\begin{abstract}
The power system is a complex nonlinear system composed of many dynamic components that interact with each other. Failure of any component in the system will have varying degrees of effects on the entire system. From the perspective of the fragility of complex systems, the brittleness factor model and brittleness correlation factor model of power systems are established in this paper, and a method to determine the brittleness chain of power network is proposed. The experimental results show that the model can realize the identification of the component failure of the potential excitation system and prevent effectively the occurrence of cascading failure, and verify the accuracy and validity of the model.
\end{abstract}

Keywords: power systems, system brittleness, brittle correlation factor, brittleness factor

Cite This Article: Xiao Lyu, Dan Lyu, and Yuge Yang, "A Modeling Method of Power System Based on Brittleness Factor and Brittleness Correlation Factor.” American Journal of Electrical and Electronic Engineering, vol. 7, no. 2 (2019): 38-41. doi: 10.12691/ajeee-7-2-3.

\section{Introduction}

Power system is one of the most complex man-made systems known at present, and its reasonable modeling is of great significance for studying the operating mechanism of power systems [1,2]. According to the theory of complex system brittleness, the power system can be abstracted into a complex system composed of many dynamic components that interact with each other. If any component fails, it will have different effects on the entire system [3,4]. From the perspective of complex systems, the power system failure is regarded as a global process. Studying the internal connections of various components in the power system can reveal the propagation law of faults. By studying the intrinsic relationship between component failure and power system cascading failure, it can reveal the action mechanism of component failure to trigger the cascading failure of power system, and provide a theoretical basis for preventing the occurrence of cascading failure.

Power system failure can be regarded as the process of brittle propagation of power system component failure in the system. The faulty component that can stimulate the system brittleness and cause the power system fault is called the malfunction of the power system. The power system fault is the result of the brittleness of the power system being excited $[5,6]$. The principle of brittle propagation is shown in Figure 1. The brittle propagation path of the power system is the brittle chain of the power system.

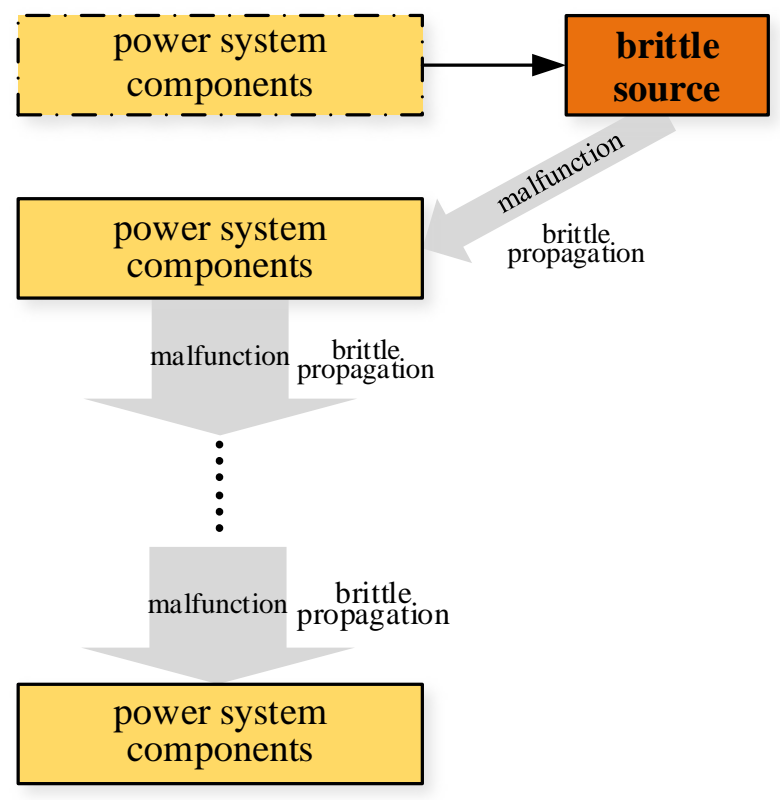

Figure 1. Brittleness propagation principle

The brittleness theory of complex systems in this paper are applied to establish the brittleness factor model and brittleness correlation factor model of power system components. The process of brittleness and brittle chain formation of power system components is described. The intrinsic link between component failure and power system cascading failures is revealed, and when any component fails, its effect on the catastrophic failure of the power system is shown. A method for determining the 
brittle chain of power system is proposed in this paper. The method is used to find the brittle propagation path of the power system, identify the weak parts of the power system, and provide an intuitive basis for preventing the cascading failure of the power system.

\section{Power System Brittleness Modeling}

\subsection{Brittleness Factor}

The power system in normal operation can resist most disturbances because its components have a certain stability margin. The size of the component's stability margin determines the type and extent of disturbance that the system can resist. Accurate identification of the brittle source of the power system can prevent the occurrence of cascading failures more easily and accurately. Therefore, the brittleness factor of the component is defined as $D$ in order to characterize the degree of brittleness of the power system after any component failure. It is known that the ratio $\lambda_{\alpha}$ of the actual capacity of component $\alpha$ to the rated capacity is:

$$
\lambda_{\alpha}=\frac{P_{\mathrm{a}}}{P_{\mathrm{r}}} .
$$

After component $\alpha$ fails, the power change of component $\beta$ is $S_{\alpha \beta}$, then the brittleness factor $D_{\alpha}$ of component $\alpha$ is:

$$
\begin{gathered}
D_{\alpha}=j \cdot \lambda_{\alpha} \sum_{\alpha=1}^{n}\left(\frac{S_{\alpha \beta}}{P_{\alpha, \mathrm{h}}}\right)^{2}+i \sum_{\alpha=1}^{n}\left[X_{\alpha}\left(\frac{S_{\alpha \beta}}{P_{\alpha, \mathrm{h}}}\right)^{2}\right] \\
\alpha=1,2, \cdots, n .
\end{gathered}
$$

$P_{\alpha, \mathrm{h}}$ is the active power thermal stability limit of component $\alpha ; X_{\alpha}$ is the reactance value of component $\alpha ; n$ is the component number; $j$ is the component $\alpha$ overload weight, and $i$ is the component $\alpha$ voltage limit weight, respectively, which are selected according to the actual system.

It can be seen from equation (2) that the larger the brittleness factor $D_{\alpha}$ is, the greater the degree of influence inserted on the operation of the power system after the failure of the component $\alpha$, and the greater the possibility of exciting the system for brittleness and forming a brittle chain. Therefore, when the operating state of the power system changes due to disturbance, the brittleness factor of each component can be calculated. According to the brittleness factor of each component, the fault prevention can be strengthened for components that may excite the brittleness of the system, which can effectively prevent the occurrence of cascading failures.

\subsection{Brittleness Correlation Factor}

A cascading failure is a chain reaction in which any component in the system fails or is in an abnormal state of operation due to a failure of any component in the system. This can also be understood as the formation process of the system fragile chain. The brittle source of the power system is the initial component that forms the brittle chain of the system, and the result of the brittleness of the power system is the occurrence of cascading failure $[7,8]$.

In the process of brittle propagation, the failure of the brittle source will cause another component failure in the system. This paper uses the brittle correlation factor to characterize the relationship between other components in the system and the brittle source. Assume that the active power of component $\alpha$ is $P_{\alpha, 0}$, the active power thermal stability limit is $P_{\alpha, \mathrm{h}}$, and the thermal stability margin $m_{\alpha}$ of other components when the component $\alpha$ activated as a brittle source is:

$$
\begin{aligned}
& m_{\alpha}=\frac{P_{\alpha, \mathrm{h}}-P_{\alpha, 0}}{P_{\alpha, \mathrm{h}}} \\
& \alpha=1,2, \cdots, n-1 .
\end{aligned}
$$

After the brittle source component $\alpha$ fails, the power transfer causes the power variation of the other component $\beta$ to be $S_{\alpha \beta}$, and the brittle source component $\alpha$ itself bears the active power $P_{\alpha}$, then the component $\alpha$ has a brittleness correlation factor $C_{\alpha \beta}$ for the component $\beta$ defined as:

$$
C_{\alpha \beta}=S_{\alpha \beta} P_{\alpha}-S_{\alpha \beta} P_{\alpha} m_{\alpha}
$$

Define the overload factor $\varphi_{\alpha}$ of component $\alpha$ as:

$$
\varphi_{\alpha}=S_{\alpha \beta} P_{\alpha} m_{\alpha}
$$

If component $\alpha$ in the power system is overloaded, its overload factor $\varphi_{\alpha}<0$, then the greater its absolute value, the more serious the overload.

Since the brittleness between system components under normal operating conditions is weak, so when $\varphi_{\alpha}>0$, the failure of component $\alpha$ does not cause the next component to be overloaded, and component $\alpha$ does not become a source of brittleness, which is only a disturbance to the system.

When $\varphi_{\alpha}<0$, if the failure of component $\alpha$ causes the other components to be overloaded, the source of brittleness is excited, causing a chain reaction in the power system which is determined by the brittle correlation.

In summary, the cascading failure leads to the process of large-scale power outage or even system collapse, which can be seen as the process of brittle chain formation in the power system, that is, the disturbance in the system is increasing, and the brittle correlation factor between components is increasing and the brittle source is activated.

\subsection{Method for Determining Brittle Chain of Power System}

The brittleness factor of the component characterizes the degree of influence on the brittleness of the excited power system when the component fails. The brittle correlation factor between components is the basis for finding the brittle propagation path.

Therefore, by calculating the brittleness factor of the component and the brittle correlation coefficient between the components, determining the brittle chain of the power system and identifying the weak link of the system can effectively avoid the occurrence of cascading failure. 
The method of determining the brittle chain of the power system is as shown in Figure 2.

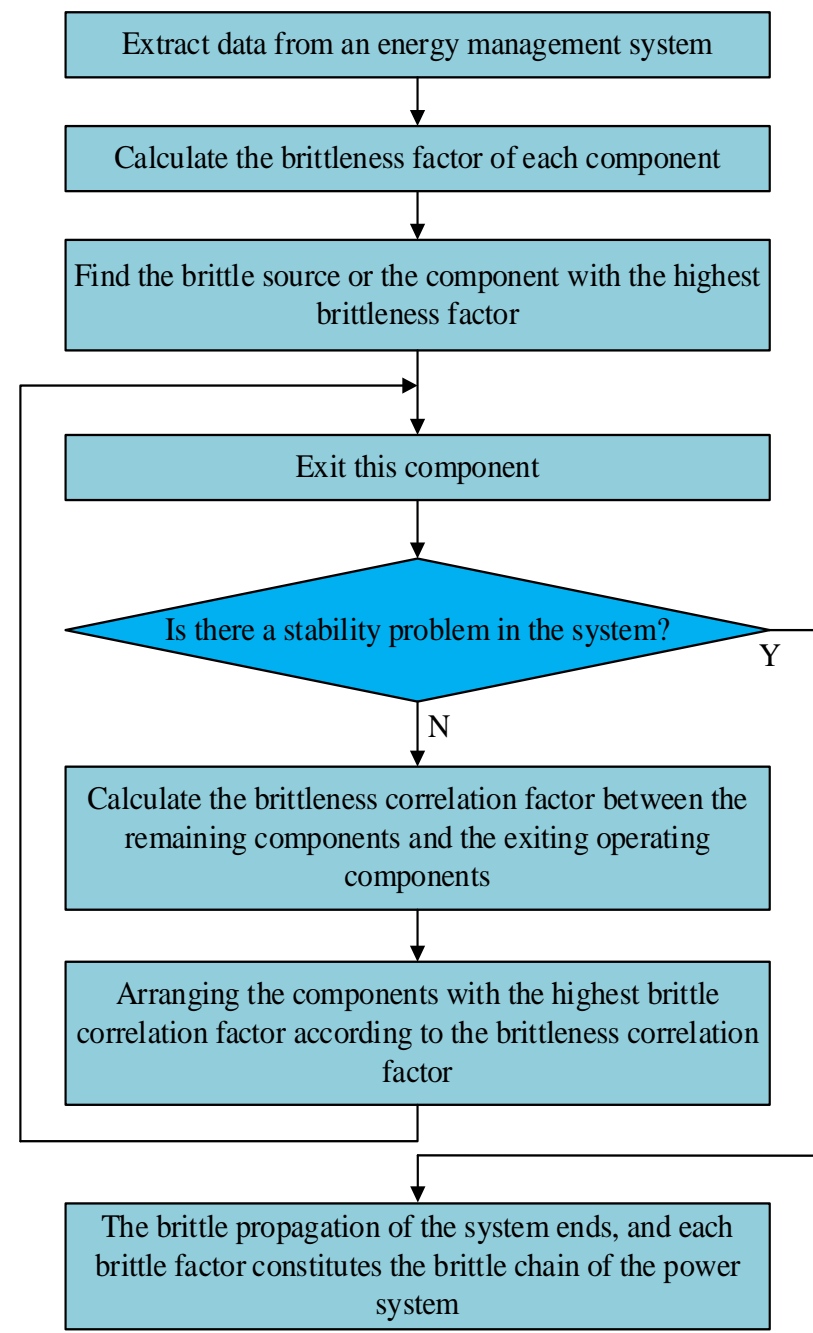

Figure 2. Method of determining the brittleness chain

(1) Considering the actual operation, the data is first extracted from the energy management system, and then the brittleness factor of each component in different power flow modes is calculated according to equation (1). If a component's failure and trip can cause other components to overload, then it can be determined that the component is a source of brittleness. If the source of brittleness cannot be determined, then the component with the highest brittleness factor should be marked.

(2) After the brittle source element or the element with the highest brittleness factor is taken out of operation, the brittleness correlation factor between the brittle source element and the remaining element is calculated. According to the calculated brittleness correlation factor, sorted by size, and marked the component with large brittle correlation factor.

(3) The larger brittleness factor selected in step (2) is used as the new brittle source, and the calculation of steps (1) and (2) is repeated until the system has a stability problem and the operation is terminated $[9,10]$.

(4) In the power system, the fault element from the first brittle source or the element with the highest brittleness factor to the last system instability problem forms a plurality of brittleness factors, which together constitute the brittle chain of the power system.

\section{Case Analysis}

This section takes the large load from November to December in northwestern China as an example. It only discusses the fault of the line, and considers the faulty line as a brittle source. Thereby, the determination of the brittle chain of the power system is performed, and the cascading failure of the power system is analyzed. The main backbone of the power system in the region is shown in Figure 3.

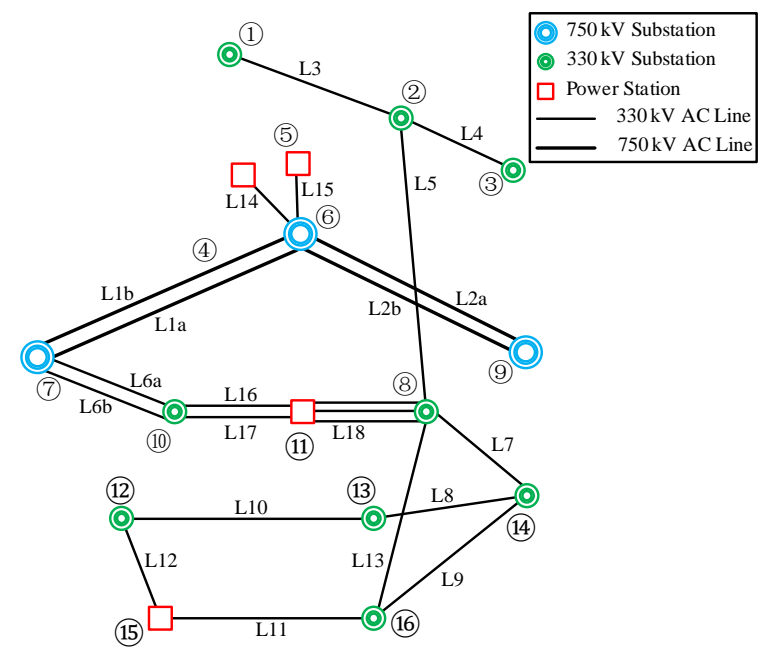

Figure 3. A section of the network wiring diagram of the power system

The power system in the region adopts the operation mode of 4,500 MW from the West-East Power Transmission, and the power flow trend of some lines is shown in Table 1.

Table 1. Power Flow Trend of Some Transmission Lines

\begin{tabular}{|c|c|c|}
\hline Line & $\begin{array}{c}\text { Sending Port Active } \\
\text { Power /MW }\end{array}$ & $\begin{array}{c}\text { Receiving Port Active } \\
\text { Power /MW }\end{array}$ \\
\hline $330 \mathrm{kV} \mathrm{L7}$ & 491.3 & 479.2 \\
\hline $330 \mathrm{kV} \mathrm{L8}$ & 226.9 & 221.3 \\
\hline $330 \mathrm{kV} \mathrm{L9}$ & 278.8 & 272.3 \\
\hline $330 \mathrm{kV} \mathrm{L4}$ & 185.6 & 182.3 \\
\hline $750 \mathrm{kV} \mathrm{L2a}$ & 1660.1 & 1660.1 \\
\hline $750 \mathrm{kV} \mathrm{L2b}$ & 1653.4 & 1653.4 \\
\hline
\end{tabular}

Consider the reactive reserve and dynamic reactive power compensation of the generator in the system. In equation (2), $j$ takes 1 and $i$ takes 0.1 . According to the calculation results, four lines with brittleness factors from large to small are listed, as shown in Table 2. When the power system in the region runs in the 4,500 MW mode of the West-East Power Transmission, the simulation calculations show that the system satisfies the $\mathrm{N}-1$ check, so the circuit brittleness will not be triggered after the line faults in Table 2 are tripped.

Table 2. Four Lines with the Largest Brittle Factors

\begin{tabular}{|c|c|c|}
\hline Faulty Line & Brittleness Factor $D$ & $\begin{array}{c}\text { Line Load Rate During } \\
\text { Normal Operation }\end{array}$ \\
\hline $750 \mathrm{kV} \mathrm{L2a(b)}$ & 1.283 & 0.338 \\
\hline $330 \mathrm{kV} \mathrm{L9}$ & 0.993 & 0.404 \\
\hline $330 \mathrm{kV} \mathrm{L3}$ & 0.859 & 0.692 \\
\hline $330 \mathrm{kV} \mathrm{L10}$ & 0.807 & 0.569 \\
\hline
\end{tabular}


According to Table 2, the $750 \mathrm{kV}$ line 2a with the highest brittleness factor is used as the system disturbance, and the next system brittleness process analysis is performed. After the $750 \mathrm{kV}$ line $2 \mathrm{a}$ is taken out of operation, the calculated brittleness correlation factors of other lines are shown in Table 3.

Table 3. Brittleness Correlation Factor of Other Lines

\begin{tabular}{|c|c|c|}
\hline Sort & Brittleness Source & Brittleness Correlation Factor $M$ \\
\hline 1 & $750 \mathrm{kV} \mathrm{L2b}$ & 9.952 \\
\hline 2 & $330 \mathrm{kV} \mathrm{L10}$ & 5.773 \\
\hline 3 & $330 \mathrm{kV} \mathrm{L9}$ & 5.012 \\
\hline 4 & $330 \mathrm{kV} \mathrm{L8}$ & 4.928 \\
\hline 5 & $330 \mathrm{kV} \mathrm{L4}$ & 4.901 \\
\hline 6 & $330 \mathrm{kV} \mathrm{L6(a)}$ & 4.835 \\
\hline
\end{tabular}

It can be seen from Table 3 that after the $750 \mathrm{kV}$ line $2 \mathrm{a}$ exits the operation, the $750 \mathrm{kV}$ line $2 \mathrm{~b}$ fault trip has the most serious impact on the power system. Therefore, the $750 \mathrm{kV}$ line $2 \mathrm{~b}$ is selected as the new brittle source and is taken out of operation. The above process is repeated, and the new brittle source obtained according to the calculation result is $750 \mathrm{kV}$ line $2 \mathrm{~b}, 330 \mathrm{kV}$ line 10,330 $\mathrm{kV}$ line 9, $330 \mathrm{kV}$ line 8, $330 \mathrm{kV}$ line 4 and $330 \mathrm{kV}$ line $6 \mathrm{a}$. Also, after the $330 \mathrm{kV}$ line $6 \mathrm{a}$ is taken out of operation, the system will become unstable and terminate the operation. Therefore, it can be determined that all the above lines are brittle units in the power system of this example, and together constitute the fragile chain of the system.

In this paper, the method for determining the brittle chain of power system greatly reduces the amount of calculation, can quickly determine the source of brittleness and find the brittle propagation path composed of brittle factors, identify the weak links of the system, and make the goal of preventing cascading failures simple and clear.

\section{Conclusion}

Based on the analysis of the brittleness of complex power systems, this paper expounds the propagation mechanism of cascading failures from the perspective of brittleness-related components. The brittleness factor model and the brittle correlation factor model of the power system components are established. A method for determining the brittle chain of power system is proposed. The specific conclusions are as follows:

(1) From the perspective of the brittleness of the power system, it is stated that the power system fault can be understood as the process of brittle propagation of system component faults in the system. The component that causes the fault is the brittle source of the system, and the fault of the power system is the result of the brittleness of the power system being excited.
(2) By finding the brittle source under different operating modes of the power system and calculating the brittle correlation coefficient between the component and the brittle source, the ability of the system to withstand the disturbance can be analyzed. And it can determine the brittle chain of the system and identify the weak links of the system, thus playing a positive role in preventing cascading failures.

(3) In the analysis of the example, the brittle chain judgment method was applied, and all the brittleness factors were successfully found and the weak links were identified. The feasibility and practicability of the judgment method were verified.

However, the analysis and calculation of this example is relatively simple. Only the faulty line is discussed. The other power system components have become a brittle source due to the failure, and further research is needed.

\section{References}

[1] Hongjie Jia, Dan Wang, Xiandong Xu and Xiaodan Yu, "Research on Some Key Problem Related to Integrated Energy Systems" Automation of Electric Power Systems, 2015. (07): 198-207.

[2] Hongliang Wu, Ershun Du, Kun Men and Liu Yang, "A Low-Carbon Oriented Energy-Saving and Economic Operation Evaluation System" Power System Technology, 2015. (05): 1179-1185.

[3] Ran Cao, Hongzhang Jin, Liyuan Feng and $\mathrm{Xu} \mathrm{Xu,} \mathrm{"Cascading}$ Failure Prevention of Complex Systems Based on Brittleness Entropy Game" High Technology Letters, 2013. (04): 422-428.

[4] Hong Zhang, Changzhen $\mathrm{Hu}$ and Xiaojun Wang, "Brittleness Analysis and Important Nodes Discovery in Large Time-Evolving Complex Networks" Journal of Shanghai Jiaotong University (Science), 2017. (01): 50-54.

[5] Yijia Cao, Yanru Chen, Lihaung Cao and Yudong Tan, "Prospects of Studies on Application of Complex System Theory in Power Systems" Proceedings of the CSEE, 2012. (19): 1-9+178.

[6] Huayi Zhang and Jingjing Zhang, "Brittle Risk Entropy Based Model for Forcasting Power System Cascading Failures" Proceedings of the CSU-EPSA, 2015. (04): 39-43.

[7] Yingying Wang, Yi Luo, Guangyu Tu and Pei Liu, "Correlation Model of Cascading Failures in Power System" Transactions of China Electrotechnical Society, 2012. (02): 204-209.

[8] Hongzhang Jin, Qi Wei and Jian Guo, Brittleness Theory and Application of Complex Systems, Northwestern Polytechnic University Press, Xi An, 2010.

[9] Yinbiao Shu, Yong Tang and Huadong Sun, "Research on Power System Security and Stability Standards" Proceedings of the CSEE, 2013. (25): 1-9.

[10] Yong Tang, "Framework of Comprehensive Defense Architecture for Power System Security and Stability" Power System Technology, 2012. (08):1-5.

[11] Zhu Z Y, Chen R P, "The Biggest Collapse Path of Brittleness of Ship Power System Based on Chaos Particle Swarm Optimization" Journal of Wuhan University of Technology, 2013. 35(3):68-72.

[12] Yin Yang, Qingwen Ren, Ying Tian and Yao Xiong, "Risk Analysis for A Cascade Reservoir System Using the Brittle Risk Entropy Method" Science China (Technological Sciences), 2016. (06):882-887. 